\title{
sTREM-1 predicts mortality in hospitalized patients with infection in a tropical, middle- income country
}

Shelton W. Wright ${ }^{1}$, Lara Lovelace-Macon ${ }^{2}$, Viriya Hantrakun ${ }^{3}$, Kristina E. Rudd ${ }^{4}$, Prapit Teparrukkul ${ }^{5}$, Susanna Kosamo ${ }^{2}$, W. Conrad Liles ${ }^{6}$, Direk Limmathurotsakul ${ }^{3,7}$ and T. Eoin West ${ }^{2,8^{*}}$ (D)

\begin{abstract}
Background: Few studies of biomarkers as predictors of outcome in infection have been performed in tropical, low- and middle-income countries where the burden of sepsis is highest. We evaluated whether selected biomarkers could predict 28-day mortality in infected patients in rural Thailand.

Methods: Four thousand nine hundred eighty-nine adult patients admitted with suspected infection to a referral hospital in northeast Thailand were prospectively enrolled within $24 \mathrm{~h}$ of admission. In a secondary analysis of 760 patients, interleukin-8 (IL-8), soluble tumor necrosis factor receptor 1 (sTNFR-1), angiopoietin-1 (Ang-1), angiopoietin2 (Ang-2), and soluble triggering receptor expressed by myeloid cells 1 (sTREM-1) were measured in the plasma. Association with 28-day mortality was evaluated using regression; a parsimonious biomarker model was selected using the least absolute shrinkage and selection operator (LASSO) method. Discrimination of mortality was assessed by receiver operating characteristic curve analysis and verified by multiple methods.
\end{abstract}

Results: IL-8, sTNFR-1, Ang-2, and STREM-1 concentrations were strongly associated with death. LASSO identified a three-biomarker model of STREM-1, Ang-2, and IL-8, but sTREM-1 alone provided comparable mortality discrimination $(p=0.07)$. sTREM-1 alone was comparable to a model of clinical variables (area under receiver operating characteristic curve [AUC] 0.81, 95\% confidence interval [CI] 0.77-0.85 vs AUC 0.79, 95\% Cl 0.74-0.84; $p=$ 0.43). The combination of STREM-1 and clinical variables yielded greater mortality discrimination than clinical variables alone (AUC 0.83, 95\% Cl 0.79-0.87; $p=0.004$ ).

Conclusions: STREM-1 predicts mortality from infection in a tropical, middle-income country comparably to a model derived from clinical variables and, when combined with clinical variables, can further augment mortality prediction.

Trial registration: The Ubon-sepsis study was registered on ClinicalTrials.gov (NCT02217592), 2014.

Keywords: Sepsis, Soluble triggering receptor expressed by myeloid cells 1, sTREM-1, Low- and middle-income countries, LMIC

\footnotetext{
* Correspondence: tewest@uw.edu

2Division of Pulmonary, Critical Care and Sleep Medicine, Department of Medicine, University of Washington, Seattle, WA 98195, USA

${ }^{8}$ University of Washington, Box 359640, 325 Ninth Ave., Seattle, WA 98104,

USA

Full list of author information is available at the end of the article
}

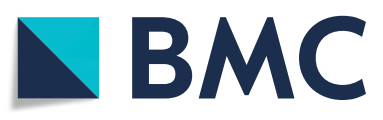

(c) The Author(s). 2020 Open Access This article is licensed under a Creative Commons Attribution 4.0 International License, which permits use, sharing, adaptation, distribution and reproduction in any medium or format, as long as you give appropriate credit to the original author(s) and the source, provide a link to the Creative Commons licence, and indicate if changes were made. The images or other third party material in this article are included in the article's Creative Commons licence, unless indicated otherwise in a credit line to the material. If material is not included in the article's Creative Commons licence and your intended use is not permitted by statutory regulation or exceeds the permitted use, you will need to obtain permission directly from the copyright holder. To view a copy of this licence, visit http://creativecommons.org/licenses/by/4.0/. The Creative Commons Public Domain Dedication waiver (http://creativecommons.org/publicdomain/zero/1.0/) applies to the data made available in this article, unless otherwise stated in a credit line to the data. 


\section{Background}

Sepsis, defined as organ dysfunction from a dysregulated immune response to infection, is a feared complication of infection and a major cause of death worldwide. Low- and middle-income countries (LMICs) - where etiologies, host factors, and clinical management may differ from highincome settings-are particularly impacted $[1,2]$. Sepsisrelated mortality in tropical Southeast Asia is especially high and related to a heterogenous group of causes [2,3].

Early and accurate assessment of the sepsis-related clinical trajectory is imperative, especially in settings with developing emergency transportation infrastructure and limited tertiary healthcare centers [1]. Identifying patients at the lowest and highest risk of deterioration permits accurate triage and judicious use of scarce resources. Thailand is an upper middle-income country with a tropical climate [4]. The northeast region of Thailand has diverse causes of sepsis, including leptospirosis, melioidosis, dengue, scrub and murine typhus, and malaria [3, 5-7]. In one referral hospital in this region, where some critical care resources are limited, we have reported that septic patients with respiratory failure and shock are commonly managed on general medical wards [8]. Optimizing prediction of outcomes from sepsis in environments such as this is essential.

Clinical scoring systems, such as the Sequential Organ Failure Assessment (SOFA) score, have been proposed as methods for predicting sepsis-related outcomes, including mortality [9]. Furthermore, modified versions of the SOFA score have been successful in predicting outcomes in LMICs, including parts of Southeast Asia [10, 11]. Clinical scoring systems, including SOFA, are frequently based on gradations of advanced cardiorespiratory support. However, in many LMICs, critically ill patients may be treated outside traditional intensive care units or without advanced support capabilities, potentially making the application of such clinical scores challenging [10].

Biomarkers, particularly those measurable in the peripheral venous blood, have become increasingly popular as complementary or alternative methods for predicting sepsis-related outcomes. Inflammatory markers such as procalcitonin and C-reactive protein have been proposed as both diagnostic and prognostic biomarkers in sepsis, although their performance in tropical Southeast Asia is inconsistent $[12,13]$. Other inflammatory markers such as soluble triggering receptor expressed by myeloid cells 1 (sTREM-1), interleukin-8 (IL-8), and soluble tumor necrosis factor receptor 1 (sTNFR-1) have shown promise, mostly in high-resource settings with temperate climates, in predicting sepsis-related mortality $[14,15]$. Similarly, biomarkers of endothelial quiescence and activation, including angiopoietin-1 (Ang-1) and angiopoietin-2 (Ang2), have also been reported to be accurate predictors of sepsis outcome [16]. However, the external validity of the performance of these biomarkers in predicting sepsis outcomes in under-resourced tropical countries-where the etiologic pathogens, host characteristics, and clinical management strategies may be distinct-is not established [1].

In this study, we reviewed the existing literature to identify five candidate biomarkers with compelling predictive evidence in populations with sepsis. We tested the hypothesis that these biomarkers could predict 28day mortality in adults hospitalized with infection of various etiologies and at risk for sepsis in a tropical setting in rural Thailand.

\section{Methods}

\section{Study design, setting, and participants}

Subjects aged 18 years or older admitted to Sunpasitthiprasong Hospital in Ubon Rachathani, Thailand, with suspected infection were prospectively enrolled between 2013 and 2017 and have been reported previously [7, 10, 11]. Enrollment prospectively occurred within $24 \mathrm{~h}$ of admission to the study hospital if subjects possessed at least three documented systemic manifestations of infection, as proposed by the 2012 Surviving Sepsis Campaign [17]. Plasma samples were obtained at the time of enrollment. As Sunpasitthiprasong Hospital is a referral center, many patients are transferred from other hospitals in the region, and these patients differ from patients who present to the study hospital emergency department (ED) by clinical characteristics and outcome [11]. Transfer from another facility was therefore considered as a possible effect modifier of the relationship between biomarker and outcome, and transfer status was included as an interaction term in the regression models. Sample size estimates were based on the presumption of an interaction and the need for stratification of the cohort by transfer status. Assumptions were based on the published area under the receiver operating curve (AUC) for mortality discrimination by SOFA score and on the distribution of plasma IL-8 concentrations in a cohort of melioidosis patients from northeast Thailand $[14,18]$. Analysis of 380 patients in each stratum would yield 99\% power to detect improvement in AUC relative to an AUC of 0.75 , assuming $10 \%$ non-survivors, log mean cytokine (variance) of $1.5(0.3)$ in non-survivors, log mean cytokine (variance) of $1.0(0.3)$ in survivors, and alpha 0.05 [19]. Three hundred eighty patients were selected by random sampling from the 551 patients presenting initially to the Sunpasitthiprasong ED, and 380 patients were randomly selected from the 3240 individuals transferred to the study hospital within $24 \mathrm{~h}$ of initial presentation. The plasma was available, and 28-day mortality outcome was known, for all 760 subjects selected.

\section{Clinical definitions}

A modified Sequential Organ Failure Assessment (modified SOFA) score was calculated for all subjects at the 
time of enrollment, given the absence of some data points such as inotrope and vasopressor agent doses and partial pressure of oxygen in arterial blood $\left(\mathrm{PaO}_{2}\right)$. This modified SOFA score has been previously described [11]. For the cardiovascular component of the SOFA score, 2 out of 4 points were given for receipt of dobutamine or dopamine and 3 out of 4 points were given for receipt of epinephrine or norepinephrine. For the respiratory component of the SOFA score, 2 out of 4 points were given if advanced respiratory support (endotracheal tube or mechanical ventilation) was utilized but arterial blood gas results were not available.

\section{Biomarker assays}

Plasma concentrations of IL-8, sTNFR-1, Ang-1, Ang-2, and sTREM-1 were measured using an electrochemiluminescence multiplex assay (Meso Scale Discovery, Rockville, MD), as previously described [20]. Samples were diluted due to the sensitivity of the assays, and upper and lower limits of detection were determined by the manufacturer's software.

\section{Biomarker selection, model development, and analysis}

A literature review was performed of biomarkers as predictors of mortality in sepsis. Five biomarkers (IL-8, sTNFR-1, Ang-1, Ang-2, and sTREM-1) were identified as having strong mortality discrimination in diverse populations with sepsis, primarily in high-resource settings [21-25]. After biomarker quantification, an Ang-2:Ang-1 ratio was calculated. Differences in biomarker concentrations between the two groups were evaluated using the Mann-Whitney $U$ test. Biomarkers were $\log _{10}$-transformed, and associations with 28-day mortality were evaluated using logistic regression. Prior to further analyses, tests of interaction were performed to evaluate the change in biomarker prediction of mortality by transfer status. Candidate models of mortality prediction were initially developed using pre-specified clinical variables including age, sex, Charlson Comorbidity Index, transfer status, a modified SOFA score, and the individual biomarker analytes. All models were assessed for the goodness of fit using the Hosmer-Lemeshow chi-square analysis. Receiver operating characteristic curve analysis was performed to evaluate mortality discrimination. To simplify the prediction model to the fewest number of variables possible, all five analytes and Ang-2:Ang-1 were subjected to logistic regression analysis by the least absolute shrinkage and selection operator (LASSO) methodology in which lambda was selected by the Akaike Information Criterion; the selected biomarkers were confirmed using the largest lambda within one standard error of the minimal mean squared prediction error based on 10-fold internal cross-validation [26, 27].
The LASSO-selected analytes and clinical variables were evaluated as predictors of 28-day mortality by creating logistic regression models and comparing AUCs to further refine the model. Subsequently, several methods were employed to verify discrimination. (1) Optimismcorrected AUCs were generated by an internal validation of 1000 replication sets by bootstrapping [28]. (2) Discrimination ability was further assessed using integrated discrimination improvement analysis (IDI) [29, 30]. (3) Nested models were subsequently compared using the likelihood ratio (LR) test [31]. (4) Finally, the net benefit of the combined biomarker and clinical variable model over the clinical variable model alone was assessed using a decision curve analysis [32]. Analyses were performed using Stata/SE version 14.2 (College Station, TX). Twosided $p$ values $<0.05$ were considered statistically significant.

\section{Results}

\section{Clinical characteristics}

The clinical characteristics of the sampled cohort are shown in Table 1. The median age was 59 years (interquartile range (IQR) 41-73), and the median Charlson Comorbidity Index score was 2 (IQR $0-4$ ). On enrollment, the median modified SOFA score was 3 (IQR 1$5)$. The 28 -day mortality rate was $14 \%$. The clinical characteristics of the patients stratified by initial ED presentation or transfer from another facility can be seen in see Additional file 1.

Biomarker concentrations in survivors and non-survivors IL-8, sTNFR-1, Ang-1, Ang-2, and sTREM-1 concentrations measured in enrollment plasma samples were

Table 1 Patient characteristics

\begin{tabular}{ll}
\hline Characteristics & Cohort $(n=760)$ \\
\hline Demographics & \\
Age in years, median (IQR) & $401(51-73)$ \\
Male sex, N (\%) & $401)$ \\
Pre-existing conditions & $2(0-4)$ \\
Charlson Comorbidity Index, median (IQR) & $157(21)$ \\
Diabetes, N (\%) & $18(2)$ \\
Chronic liver disease, N (\%) & $85(11)$ \\
Chronic kidney disease, N (\%) & $47(6)$ \\
Chronic cardiovascular disease, N (\%) & $60(8)$ \\
Chronic lung disease, N (\%) & $17(2)$ \\
Cancer, N (\%) & $9(1)$ \\
HIV, N (\%) & $3(1-5)$ \\
Modified SOFA score, median (IQR) & $492(65)$ \\
Subjects with modified SOFA score $\geq 2, N(\%)$ & $110(14)$ \\
Died within 28 days, N (\%) &
\end{tabular}


compared in survivors and non-survivors to 28-days (Table 2). Concentrations of IL-8, sTNFR-1, Ang-2, and sTREM-1, and the Ang-2:Ang-1 ratio were significantly lower in survivors compared to non-survivors (all $p<$ 0.001). There was no difference in concentrations of Ang-1.

\section{Association of biomarkers with death}

The association between $\log _{10}$-transformed biomarker concentration and death was analyzed using logistic regression. Given the possibility of effect modification by transfer status, an interaction between each biomarker and transfer status on the association with death was first assessed in the models (see Additional file 2). A significant interaction with transfer status was found only for Ang-2 $(p=0.03)$. In the full cohort, increased concentrations of IL-8, sTNFR-1, sTREM-1, and Ang-2: Ang-1 each were significantly associated with death (all $p<0.001$; Table 3). The association of Ang-2 with death was separately evaluated in the set of non-transferred patients and in the set of transferred patients; increased concentrations of this biomarker were significantly associated with death in both sets $(p<0.001$ in both sets; see Additional file 3). However, in models adjusted for age, sex, Charlson Comorbidity Index, and modified SOFA score, no significant interaction of any biomarker with transfer status was found (see Additional file 2). In the full cohort, IL-8, sTNFR-1, Ang-2, sTREM-1, and Ang2:Ang-1 remained associated with death when the models were adjusted for age, sex, transfer status, Charlson Comorbidity Index, and modified SOFA score at enrollment (all $p<0.001$, Table 3). The biomarker with the highest point estimate for the odds of death was sTREM-1 (adjusted odds ratio (OR) 11.2 for each $\log _{10}$ increase in sTREM1, 95\% confidence interval (CI) 4.925.9). All models had appropriate goodness of fit as assessed by the Hosmer-Lemeshow method $(p>0.05)$.

\section{Discrimination of mortality}

In order to develop the most parsimonious biomarker model to predict mortality, LASSO regression was performed on the five analytes and on the Ang-2:Ang-1
Table 3 Association of biomarkers with death at 28 days

\begin{tabular}{lllllll}
\hline Biomarker $^{a}$ & \multicolumn{2}{l}{ Unadjusted } & & \multicolumn{2}{l}{ Modified SOFA-adjusted $^{\mathrm{b}}$} \\
\cline { 2 - 3 } & OR $(95 \% \mathrm{Cl})$ & $p$ value & & OR $(95 \% \mathrm{Cl})$ & $p$ value \\
\hline IL-8 & $2.3(1.9-2.9)$ & $<0.001$ & & $1.9(1.5-2.4)$ & $<0.001$ \\
STNFR-1 & $21.3(10.6-42.8)$ & $<0.001$ & & $5.2(2.3-12.1)$ & $<0.001$ \\
Ang-1 & $1.1(0.8-1.6)$ & 0.48 & & $1.2(0.8-1.8)$ & 0.4 \\
Ang-2 & $11.3(6.6-19.3)$ & $<0.001$ & & $5.4(2.8-10.3)$ & $<0.001$ \\
Ang-2:Ang-1 & $2.2(1.7-3.0)$ & $<0.001$ & & $1.5(1.1-2.2)$ & 0.02 \\
STREM-1 & $33.0(16.3-67.0)$ & $<0.001$ & & $11.2(4.9-25.9)$ & $<0.001$
\end{tabular}

${ }^{\mathrm{a} B i o m a r k e r s ~ w e r e ~} \log _{10}$-transformed before regression; each biomarker was assessed in separate models

${ }^{\text {b} M o d e l s ~ w e r e ~ a d j u s t e d ~ f o r ~ a g e, ~ s e x, ~ t r a n s f e r ~ s t a t u s, ~ C h a r l s o n ~ C o m o r b i d i t y ~}$ Index, and modified SOFA score

ratio. This procedure selected sTREM-1, Ang-2, and IL8 (see Additional file 7). Discrimination of mortality by the LASSO-selected three-biomarker model was determined by calculating the area under the receiver operating characteristic curve (AUC) $0.83,95 \%$ CI $0.79-0.87$. Compared to each of the biomarkers alone (Table 4), the three-biomarker model was superior to both Ang-2 and IL-8 but was not significantly better than sTREM-1 (AUC $0.81,95 \%$ CI $0.77-0.85, p=0.07$ ), suggesting that sTREM-1 alone may provide comparable mortality discrimination. To evaluate how sTREM-1 performed relative to the available clinical data, a clinical variable model-comprising age, sex, Charlson Comorbidity Index, transfer status, and modified SOFA score-was developed. sTREM-1 had comparable mortality discrimination as the clinical variable model (AUC 0.81, 95\% CI $0.77-0.85$ vs AUC 0.79 , 95\% CI $0.74-0.84 ; p=0.43$; Table 5, Fig. 1). To determine whether sTREM-1 augmented clinical data, sTREM-1 was added to the clinical variable model. For the combined model, mortality discrimination (AUC 0.83, 95\% CI 0.79-0.87) was significantly greater than for the clinical variable model alone $(p=0.004)$ and was borderline better than STREM-1 alone $(p=0.05)$. Several other methods were used to verify these observations. The sTREM- 1 models and the clinical variable model demonstrated minimal bias in optimism-adjusted AUCs after bootstrap validation (see Additional file 4). Discrimination analysis by IDI and the

Table 2 Plasma biomarker concentration in survivors and non-survivors of infection

\begin{tabular}{lllll}
\hline Biomarker $(\mathrm{pg} / \mathrm{ml})$ & All, median $(\mathrm{IQR})(n=760)^{\mathrm{a}}$ & Survivors, median (IQR) $(n=650)$ & Non-survivors, median (IQR) $(n=110)$ & $p$ value \\
\hline IL-8 & $14(7-55)$ & $12(6-31)$ & $76(31-933)$ & $<0.001$ \\
sTNFR-1 & $7749(3650-17,354)$ & $6491(3408-14,346)$ & $20,571(11890-30,923)$ & $<0.001$ \\
Ang-1 & $2012(925-3874)$ & $1985(899-3835)$ & $2083(989-4028)$ & 0.58 \\
Ang-2 & $8859(4436-17,496)$ & $7751(4043-15,042)$ & $23,939(10432-42,212)$ & $<0.001$ \\
Ang-2:Ang-1 & $5(2-16)$ & $4(2-14)$ & $14(4-35)$ & $<0.001$ \\
STREM-1 & $405(241-830)$ & $361(224-655)$ & $1037(647-1727)$ & $<0.001$ \\
\hline
\end{tabular}

${ }^{a}$ For Ang-1 and Ang-2, concentrations were measured in 748 samples

${ }^{\mathrm{b}} \mathrm{p}$ value calculated using the Mann-Whitney $U$ test 
Table 4 Discrimination of mortality by LASSO-selected biomarkers

\begin{tabular}{llll}
\hline Model & AUC & $95 \% \mathrm{Cl}$ & $p$ value $^{\mathrm{a}}$ \\
\hline IL-8+Ang-2+sTREM-1 & 0.83 & $0.79-0.87$ & Ref \\
IL-8 & 0.77 & $0.73-0.81$ & 0.002 \\
Ang-2 & 0.77 & $0.73-0.82$ & 0.0002 \\
STREM-1 & 0.81 & $0.77-0.85$ & 0.07 \\
\hline
\end{tabular}

${ }^{a}$ Test of equality between AUC of listed models and LASSO-selected IL-8+Ang-2+sTREM-1 model

LR test showed significant improvement of the clinical variable model when sTREM-1 was added (IDI: $p<$ 0.001 , see Additional file 4 ; $L R=1.6 \times 10^{-9}$, see Additional file 5). Finally, decision curve analysis demonstrated an increased net benefit of the model combining clinical variables and sTREM-1 over a clinical variable model alone, most notably in the decrease in falsepositive prediction of death (see Additional files 6 and 8).

\section{STREM-1 increases with clinical severity of illness determined by modified SOFA score}

As STREM-1 alone and a clinical variable model including a modified SOFA score were comparable classifiers for the discrimination of death, we assessed the relationship between sTREM-1 levels and modified SOFA scores. STREM-1 concentrations were positively correlated with quartile of modified SOFA score (Fig. 2, $r=$ $0.53, p<0.001)$.

\section{Discussion}

In this study, we validated the performance of several biomarkers in predicting death from infection in a tropical, middle-income country. In a relatively large cohort of patients with infection admitted to a referral hospital either through the ED or by transfer from an outside facility, a single measurement of sTREM-1 within $24 \mathrm{~h}$ of admission is similar to clinical illness score models in predicting 28day mortality. Combining sTREM-1 with a clinical illness score model further improves discrimination.

The global burden of sepsis is highest-but the fewest studies of sepsis are performed-in resource-limited settings [1]. Due to significant differences in etiologies, host

Table 5 Clinical variable and sTREM-1 models of mortality prediction

\begin{tabular}{lllll}
\hline Model & AUC & $95 \% \mathrm{Cl}$ & $p$ value $^{\mathrm{b}}$ & $p$ value $^{c}$ \\
\hline Clinical variables $^{\mathrm{a}}$ & 0.79 & $0.74-0.84$ & Ref & - \\
sTREM-1 $^{\text {Clinical variables + sTREM-1 }}$ & 0.81 & $0.77-0.85$ & 0.43 & Ref \\
& $0.79-0.87$ & 0.004 & 0.05
\end{tabular}

${ }^{a}$ Clinical variables model includes age, sex, Charlson Comorbidity Index, transfer status, and modified SOFA score

${ }^{\mathrm{b}}$ Test of equality between AUC of listed models and the clinical variable model

'Test of equality between AUC of listed models and STREM- 1 model

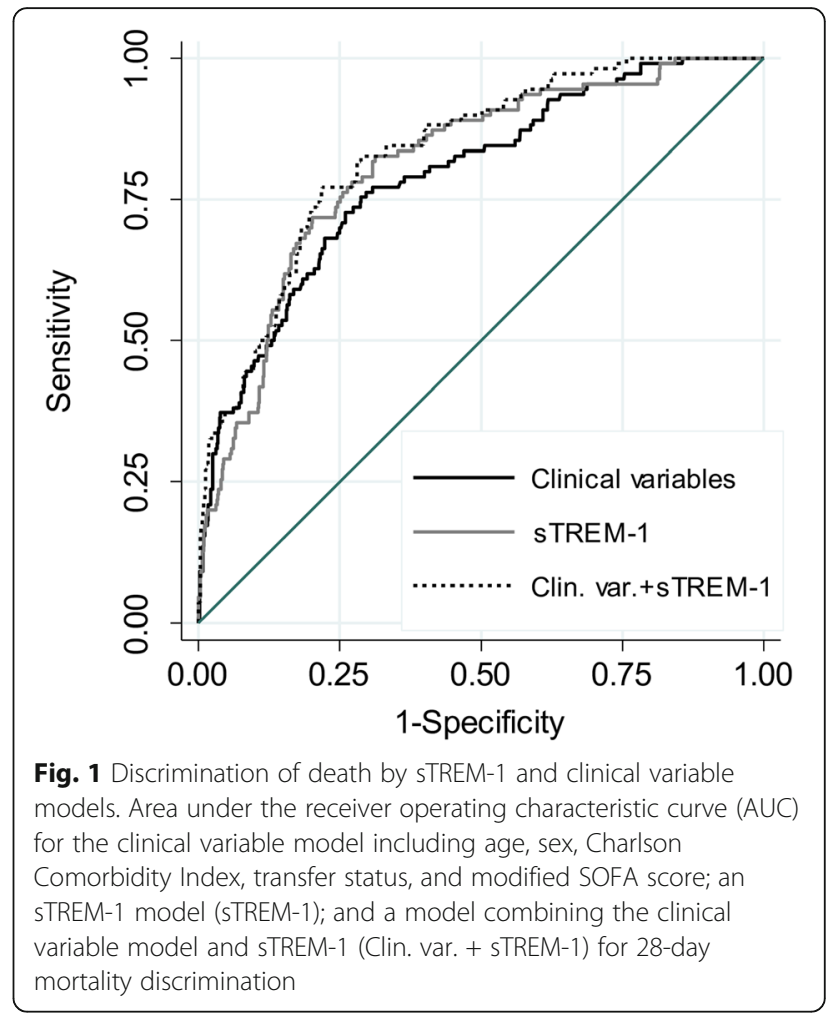

factors, and clinical management of sepsis, it is essential to perform clinical studies in these at-risk populations rather than to extrapolate from high-income settings. Several features differentiate our study population and therefore expand the validity of the selected biomarkers for the prediction of outcome in sepsis. First, in comparison with most prior studies, the etiologies of infection at the study hospital and surrounding regions are distinct and heterogeneous: Staphylococcus aureus, Escherichia coli, Burkholderia pseudomallei,

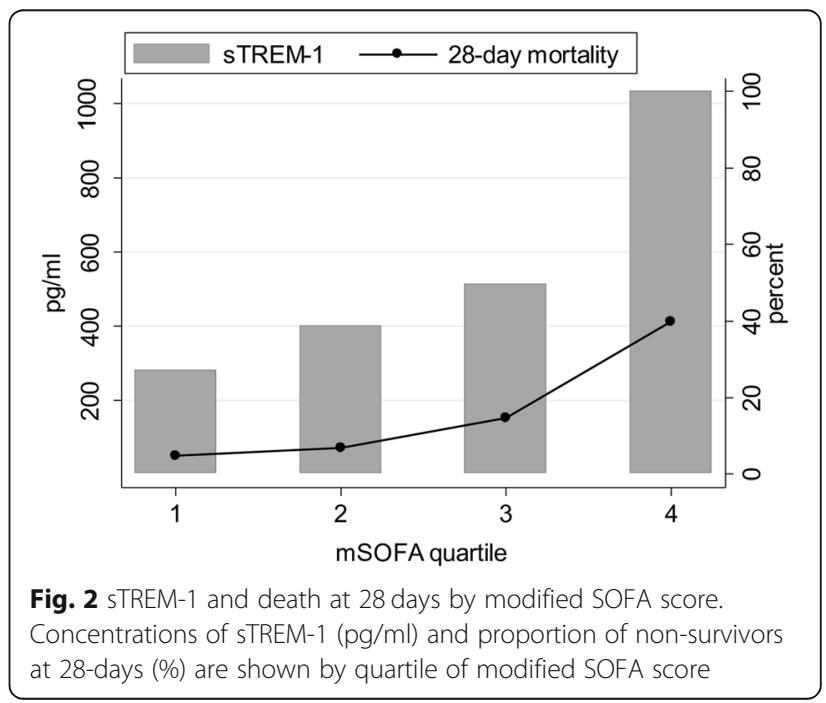


leptospirosis, scrub and murine typhus, dengue, and malaria are all described $[3,6,7,11]$. Second, in contrast to many high-resource settings, due to intensive care unit bed availability at the study hospital, management of many patients with sepsis-even with respiratory failure or shock-frequently occurs on the general medical wards [8]. Our results provide important validation of the performance of sTREM-1 in mortality prediction in these populations.

Besides STREM-1, all the other biomarkers we studied, with the exception of Ang-1, were strongly associated with 28-day mortality; moreover, discrimination of mortality by IL- 8 and Ang- 2 as measured by the AUC was 0.77 for each analyte. Others have reported that biomarkers of endothelial quiescence vs activation, such as Ang-1, Ang-2, and the ratio of Ang-2:Ang-1, are strong predictors of mortality in sepsis [16]. In Thai adults with malaria, Ang-1 levels decreased and Ang-2 levels increased with higher severity of illness [33]. As previously noted, similar patterns, as well as elevation in the Ang-2: Ang-1 ratio, have been reported in sepsis, though not in Southeast Asia or resource-limited settings [20]. Nonspecific markers of inflammation, including IL-8 and sTNFR-1, also predict sepsis-related mortality [14]. Therefore, Ang-2, IL-8, and sTNFR-1 may have additional value in sepsis outcome prediction in a resourcelimited setting and deserve further study.

Triggering receptor expressed on myeloid cells 1 (TREM-1) is a transmembrane receptor typically found on monocytes and neutrophils [34]. Activation of TREM-1 may play a role in innate inflammatory responses by modulating the secretion of proinflammatory cytokines such as IL- 8 and TNF- $\alpha$. Importantly, the activating ligand remains somewhat unclear, though during inflammation TREM-1 is released in a soluble form as sTREM-1 [35]. As sTREM-1 levels are elevated in non-infectious inflammatory states, results of its performance for diagnosis of sepsis have been mixed [15, 36, 37]. Our findings do suggest that a biomarker of innate immune activation has strong discrimination of mortality in a LMIC setting and may represent a distinct pathway compared to the other biomarkers we evaluated. Recently, in a cohort of outpatient, febrile adults in Tanzania, sTREM-1 enhanced clinical prediction of mortality [38]. Similarly, in our study, sTREM-1 was not only comparable to clinical variables in predicting mortality but enhanced mortality prediction when combined with clinical variables. However, of note, substantial differences exist between these studies, including patient setting, geographic location, rates of HIV infection, infectious etiologies, sample size, clinical scoring system, and method of biomarker measurement. Even considering these differences, our results provide additional evidence supporting the use of
sTREM-1 to augment sepsis mortality prediction in tropical, resource-limited settings, particularly in Southeast Asia.

Organ failure assessment using SOFA scores may be challenging to determine in lower-resourced areas due to the lack of available data, prompting the development of simpler clinical prediction models [10, 39, 40]. Importantly, clinical parameters have associated costs, primarily related to healthcare infrastructure [41]. However, a potential advantage of a predictive biomarker is obviating the need to calculate clinical scores of any type and, when considered within a health system, may be cost-effective. Alternatively, in settings where clinical scores can be readily calculated, a biomarker may further augment mortality prediction. In our study, a single biomarker, sTREM-1, had similar outcome prediction as a clinical model including a modified SOFA score and may be a useful substitute in remote settings where certain clinical and laboratory data are difficult to ascertain.

Certain physiologic biomarkers, such as an elevated lactate, predict mortality in LMIC settings [42]. However, the early use of sepsis biomarkers to predict clinical trajectory may inform triage and resuscitation approaches in resource-limited settings [43]. Therefore, significant attention has focused on the utility of the inflammatory markers procalcitonin and C-reactive protein, both of which are widely available but have variable performance in Southeast Asia for infection prognosis $[12,13]$. Assays for our analyzed biomarkers, including STREM-1, are not readily available in clinical laboratories. However, our results may inform the future development of a simple point-of-care assay [44, 45]. Such an assay could help guide clinical decision-making about management and referral. Further studies will need to assess whether markers of innate immune activation, like sTREM-1, have superior sepsis-related outcome prediction compared to more general inflammation markers such as procalcitonin and C-reactive protein.

Our study has several strengths. To our knowledge, this is the largest study of a population of hospitalized patients to evaluate the performance of sTREM-1 in predicting outcomes from infection and the first to examine patients in Southeast Asia. We included in our analysis patients presenting primarily to the study hospital ED as well as patients referred from a variety of surrounding hospitals in the region, increasing the external validity of our results. Sample processing, tracking, and assaying were carefully implemented. There were minimal missing data and loss to follow-up in this cohort. Finally, several methods were used to validate the performance of sTREM-1.

Our study has several potential limitations, including those previously noted. While detailed clinical history as 
well as timely blood tests was obtained, resource limitations precluded certain tests, including arterial blood gases. Similarly, dosages of vasopressor or inotropic medications were not available. These factors limited our ability to completely calculate SOFA criteria. Although we used a modified SOFA score, this potentially limits the broader applicability of our findings. The study was performed at a single public provincial hospital, although the referral system in Thailand facilitates the expeditious transfer of sick patients from over 60 surrounding hospitals to this center, resulting in a sizeable catchment area [11]. For patients transferred from another facility, the extent of care received at the transferring facilities could not be assessed. Similarly, patients admitted to non-medical services, such as surgical wards, were not enrolled and may have different characteristics than our cohort. Our biomarker analysis plan was designed to select a parsimonious model and minimize bias. Therefore, we only analyzed the mortality discrimination of LASSO-selected biomarkers. However, other biomarkers, including sTNFR-1, may have similar discrimination to those analyzed. Finally, our study occurred in a specific region of Thailand, and generalization of these results to other locations with variable genetic, economic, and infectious characteristics may be limited.

\section{Conclusions}

In summary, in a large observational study of adults in northeast Thailand hospitalized with an infection, multiple biomarkers of endothelial activation/dysfunction and innate immune activation, assayed once within $24 \mathrm{~h}$ of admission, are predictive of death. sTREM-1 has a similar mortality prediction compared to a clinical variable model that includes a modified SOFA score. sTREM-1 can further augment the clinical prediction of mortality when combined with clinical variables. These findings add new evidence to support the utility of blood biomarkers in predicting outcomes from infection and sepsis of diverse etiologies in tropical, resource-limited settings. With further study, such biomarkers may be helpful tools to guide clinical triage and resource allocation decisions for optimal patient management.

\section{Supplementary information}

Supplementary information accompanies this paper at https://doi.org/10. 1186/s12916-020-01627-5.

Additional file 1. Patient characteristics stratified by transfer status. Additional file 2. Association of biomarkers with death stratified by transfer status.

Additional file 3. Association of Ang-2 with death stratified by transfer status.
Additional file 4. Adjustment and verification of sTREM-1 predictive models.

Additional file 5. Mortality prediction using models of clinical variables with or without STREM-1.

Additional file 6 . Net benefit of adding STREM-1 to a model of clinical variables for mortality prediction.

Additional file 7: Figure S1. Biomarker selection using LASSO regression.

Additional file 8: Figure S2. Decision curve analysis.

\section{Abbreviations}

Ang-1: Angiopoietin-1; Ang-2: Angiopoietin-2; AUC: Area under the receiver operating curve; Cl: Confidence interval; ED: Emergency department; IDI: Integrated discrimination improvement analysis; IL-8: Interleukin-8; IQR: Interquartile range; LASSO: Least absolute shrinkage and selection operator; LMICs: Low- and middle-income countries; LR: Likelihood ratio; OR: Odds ratio; $\mathrm{PaO}_{2}$ : Partial pressure of oxygen in arterial blood;

SOFA: Sequential Organ Failure Assessment; sTNFR-1: Soluble tumor necrosis factor receptor 1; sTREM-1: Soluble triggering receptor expressed by myeloid cells 1; TREM-1: Triggering receptor expressed on myeloid cells 1

\section{Acknowledgements}

The authors thank the patients and staff at Sunpasitthiprasong Hospital and Mahidol-Oxford Tropical Medicine Research Unit. We also thank Mark Wurfel for his advice.

\section{Authors' contributions}

SWW designed the project, performed the biomarker measurements, analyzed the data, and wrote the first draft of the manuscript. LL-M performed the biomarker measurements. VH and DL oversaw the sample and data collection and analyzed the data. PT participated in the project design and facilitated the sample and data collection. KR participated in the data analysis. SK and WCL participated in the project design. TEW conceived the project and directed the research. All authors contributed to the writing or revision of the manuscript. All authors read and approved the final manuscript.

\section{Funding}

This work was supported by the US National Institutes of Health [grant numbers T32GM086270, T32HL007287, R01HL113382, R01Al137111] and the Wellcome Trust [090219/Z/09/Z, 101103/Z/13/Z].

\section{Availability of data and materials}

The dataset is available on reasonable request from the corresponding author (TEW).

\section{Ethics approval and consent to participate}

Written informed consent was obtained from the study participants or their representatives prior to enrollment. The studies were approved by the Sunpasitthiprasong Hospital Ethics Committee (039/2556); the Ethics Committee of the Faculty of Tropical Medicine, Mahidol University (MUTM2012-024-01); the University of Washington Institutional Review Board (42988); and the Oxford University Tropical Research Ethics Committee (OXTREC172-12).

\section{Consent for publication}

Not applicable

\section{Competing interests}

WCL has the following patents: "Biomarkers for early determination of a critical or life threatening response to illness and monitoring response to treatment thereof" (Application CA2769433 A1 - August 27, 2013: WO20131270000 A1 - September 6, 2013), "Biomarkers for malaria" (Application: WO2012016333 A1 - February 9, 2012), "Angiopoietin-1 and -2 biomarkers for infectious diseases that compromise endothelial integrity" (Application: WO2009059404 A1 - May 14, 2009; US20110008804 A1 January 13, 2011), "Biomarkers for early determination of a critical or life threatening response to illness and/or treatment response" (Application: US14/916,758 - March 4, 2016), and "Biomarkers to identify and predict severe toxicity after adoptive T cell therapy" (Application: US62/456,798 - 
February 9, 2017). All other authors declared that they have no competing interests. All authors have submitted the ICMJE Form for Disclosure of Potential Conflicts of Interest.

\section{Author details}

${ }^{1}$ Division of Pediatric Critical Care Medicine, Department of Pediatrics, University of Washington, Seattle, WA 98104, USA. '2Division of Pulmonary, Critical Care and Sleep Medicine, Department of Medicine, University of Washington, Seattle, WA 98195, USA. ${ }^{3}$ Mahidol-Oxford Tropical Medicine Research Unit, Faculty of Tropical Medicine, Mahidol University, Bangkok 10400, Thailand. ${ }^{4}$ Department of Critical Care Medicine, University of Pittsburgh, Pittsburgh, PA 15213, USA. ${ }^{5}$ Department of Internal Medicine, Sunpasitthiprasong Hospital, Ubon Ratchathani 34000, Thailand. 'Division of Allergy and Infectious Diseases, Department of Medicine, University of Washington, Seattle, WA 98195, USA. ${ }^{7}$ Department of Tropical Hygiene, Faculty of Tropical Medicine, Mahidol University, Bangkok 10400, Thailand. ${ }^{8}$ University of Washington, Box 359640, 325 Ninth Ave., Seattle, WA 98104 , USA.

\section{Received: 27 February 2020 Accepted: 11 May 2020} Published online: 01 July 2020

\section{References}

1. Rudd KE, Kissoon N, Limmathurotsakul DI, Bory S, Mutahunga B, Seymour CW, et al. The global burden of sepsis: barriers and potential solutions. Crit Care. 2018:22(232):1-11.

2. Rudd KE, Johnson SC, Agesa KM, Shackelford KA, Tsoi D, Kievlan DR, et al. Global, regional, and national sepsis incidence and mortality, 1990-2017: analysis for the Global Burden of Disease study. Lancet. 2020;6736(19):1-12.

3. Sudarmono P, Aman AT, Arif M, Syarif AK, Kosasih H, Karyana M, et al. Causes and outcomes of sepsis in Southeast Asia: a multinational multicentre cross-sectional study. Lancet Glob Health. 2017;5(2):e157-67.

4. World Bank and National Economic and Social Development Board. Thailand Northeast Economic Development Report. 2005;1-233.

5. Kanoksil M, Jatapai A, Peacock SJ, Limmathurotsakul D. Epidemiology, microbiology and mortality associated with community-acquired bacteremia in northeast Thailand: a multicenter surveillance study. PLoS One. 2013;8(1):1-9.

6. Teparrukkul P, Hantrakun V, Imwong M, Teerawattanasook N, Wongsuvan G, Day NPJ, et al. Utility of qSOFA and modified SOFA in severe malaria presenting as sepsis. PLoS One. 2019;14(10):1-13.

7. Teparrukkul P, Hantrakun V, Day NPJ, West TE, Limmathurotsakul D. Management and outcomes of severe dengue patients presenting with sepsis in a tropical country. PLoS One. 2017;12(4):1-13.

8. Rudd KE, Hantrakun V, Somayaji R, Booraphun S, Boonsri C, Fitzpatrick AL, et al. Early management of sepsis in medical patients in rural Thailand: a single-center prospective observational study. J Intensive Care. 2019;7(55):1-8.

9. Minne L, Abu-Hanna A, de Jonge E. Evaluation of SOFA-based models for predicting mortality in the ICU: a systematic review. Crit Care. 2008;12(6):1-13.

10. Rudd KE, Seymour CW, Aluisio AR, Augustin ME, Bagenda DS, Beane A, et al. Association of the quick sequential (sepsis-related) organ failure assessment (qSOFA) score with excess hospital mortality in adults with suspected infection in low- and middle-income countries. JAMA. 2018;319(21):2202-11.

11. Hantrakun V, Somayaji R, Teparrukkul P, Boonsri C, Rudd K, Day NPJ, et al. Clinical epidemiology and outcomes of community acquired infection and sepsis among hospitalized patients in a resource limited setting in northeast Thailand: a prospective observational study (Ubon-sepsis). PLoS One. 2018; 13(9):1-14.

12. van Engelen TSR, Wiersinga WJ, Scicluna BP, van der Poll T. Biomarkers in sepsis. Crit Care Clin. 2018;34(1):139-52.

13. Lubell Y, Blacksell SD, Dunachie S, Tanganuchitcharnchai A, Althaus T, Watthanaworawit $W$, et al. Performance of C-reactive protein and procalcitonin to distinguish viral from bacterial and malarial causes of fever in Southeast Asia. BMC Infect Dis. 2015:15(1):1-10.

14. Mikacenic C, Price BL, Harju-Baker S, O'Mahony DS, Robinson-Cohen C, Radella F, et al. A two-biomarker model predicts mortality in the critically ill with sepsis. Am J Respir Crit Care Med. 2017;196(8):1004-11.

15. Ríos-Toro JJ, Márquez-Coello M, García-Álvarez JM, Martín-Aspas A, RiveraFernández R, De Benito AS, et al. Soluble membrane receptors, interleukin 6, procalcitonin and $C$ reactive protein as prognostic markers in patients with severe sepsis and septic shock. PLoS One. 2017;12(4):1-18.
16. Ricciuto DR, Dos Santos CC, Hawkes M, Toltl LJ, Conroy AL, Rajwans N, et al. Angiopoietin-1 and angiopoietin-2 as clinically informative prognostic biomarkers of morbidity and mortality in severe sepsis. Crit Care Med. 2011; 39(4):702-10.

17. Seymour CW, Liu VX, Iwashyna TJ, Brunkhorst FM, Rea TD, Scherag A, et al. Assessment of clinical criteria for sepsis for the third international consensus definitions for sepsis and septic shock (sepsis-3). JAMA. 2016;315(8):762-74.

18. Kaewarpai $T$, Ekchariyawat $P$, Phunpang $R$, Wright $S$, Dulsuk $A$ Moonmueangsan $B$, et al. Longitudinal profiling of plasma cytokines in melioidosis and their association with mortality: a prospective cohort study. Clin Microbiol Infect. 2019; In press.

19. Pepe MS. The statistical evaluation of medical tests for classification and prediction. New York City: Oxford University Press; 2003. p. 302.

20. Mikacenic C, Hahn WO, Price BL, Harju-Baker S, Katz R, Kain KC, et al. Biomarkers of endothelial activation are associated with poor outcome in critical illness. PLoS One. 2015;10(10):1-13.

21. Hahn WO, Mikacenic C, Price BL, Harju-Baker S, Katz R, Himmelfarb J, et al. Host derived biomarkers of inflammation, apoptosis, and endothelial activation are associated with clinical outcomes in patients with bacteremia and sepsis regardless of microbial etiology. Virulence. 2016;7(4):387-94.

22. Jeong SJ, Song YG, Kim CO, Kim HW, Ku NS, Han SH, et al. Measurement of plasma STREM-1 in patients with severe sepsis receiving early goal-directed therapy and evaluation of its usefulness. Shock. 2012;37(6):574-8.

23. Su L, Liu D, Chai W, Liu D, Long Y. Role of sTREM-1 in predicting mortality of infection: a systematic review and meta-analysis. BMJ Open. 2016;6(5):1-8.

24. Clark DV, Banura P, Bandeen-Roche K, Liles WC, Kain KC, Scheld WM, et al. Biomarkers of endothelial activation/dysfunction distinguish sub-groups of Ugandan patients with sepsis and differing mortality risks. JCI Insight. 2019; 1:0-12.

25. Ravetti CG, Moura AD, Vieira ÉL, Pedroso ERP, Teixeira AL. STREM-1 predicts intensive care unit and 28-day mortality in cancer patients with severe sepsis and septic shock. J Crit Care. 2015;30(2):440.e7-440.e13.

26. Tibshirani R. Regression shrinkage and selection via the Lasso. J R Stat Soc Ser B. 1996:58(1):267-88.

27. Pavlou M, Ambler G, Seaman S, De lorio M, Omar RZ. Review and evaluation of penalised regression methods for risk prediction in low-dimensional data with few events. Stat Med. 2016;35(7):1159-77.

28. Penciana MJ, D'Agostino RB. Overall $C$ as a measure of discrimination in survival analysis: model specific population value and confidence interval estimation. Stat Med. 2004;23(13):2109-23.

29. Pencina MJ, D'Agostino RB Sr, D'Agostino RB Jr, Vasan RS. Evaluating the added predictive ability of a new marker: from area under the ROC curve to reclassification and beyond. Stat Med. 2008;27(2):157-72.

30. Cook NR. Quantifying the added value of new biomarkers: how and how not. Diagn Progn Res. 2018;2(1):1-7.

31. Hosmer DW, Hosmer T, Le Cessie S, Lemeshow S. A comparison of goodness-of-fit tests for the logistic regression model. Stat Med. 1997;16: 965-80.

32. Vickers AJ, Elkin EB. Decision curve analysis: a novel method for evaluating prediction models. Med Decis Mak. 2006;26(6):565-74.

33. Conroy AL, Lafferty El, Lovegrove FE, Krudsood S, Tangpukdee N, Liles WC, et al. Whole blood angiopoietin-1 and-2 levels discriminate cerebral and severe (non-cerebral) malaria from uncomplicated malaria. Malar J. 2009;8(1): $1-7$.

34. Bouchon A, Dietrich J, Colonna M. Cutting edge: inflammatory responses can be triggered by TREM-1, a novel receptor expressed on neutrophils and monocytes. J Immunol. 2000;164(10):4991-5.

35. Gibot $\mathrm{S}$, Cravoisy A. Soluble form of the triggering receptor expressed on myeloid cells 1 as a marker of microbial infection. Clin Med Res. 2004;2(3): $181-7$.

36. Gibot S, Cravoisy A, Levy B, Bene M-C, Faure G, Bollaert P-E. Soluble triggering receptor expressed on myeloid cells and the diagnosis of pneumonia. N Engl J Med. 2004;350(5):451-8.

37. Barati M, Bashar FR, Shahrami R, Zadeh MHJ, Taher MT, Nojomi M. Soluble triggering receptor expressed on myeloid cells 1 and the diagnosis of sepsis. J Crit Care. 2010;25(2):362.e1-6.

38. Richard-Greenblatt M, Boillat-Blanco N, Zhong K, Mbarack Z, Samaka J, Mlaganile T, et al. Prognostic accuracy of soluble triggering receptor expressed on myeloid cells (sTREM-1)-based algorithms in febrile adults presenting to Tanzanian outpatient clinics. Clin Infect Dis. 2019;70(7):1304-12. 
39. Moore CC, Hazard R, Saulters K, Ainsworth J, Adakun SA, Amir A, et al. Derivation and validation of a universal vital assessment (UVA) score: a tool for predicting mortality in adult hospitalised patients in sub-Saharan Africa. BMJ Glob Health. 2017:2(2):1-12.

40. Haniffa R, Mukaka M, Munasinghe SB, De Silva AP, Jayasinghe KSA, Beane A, et al. Simplified prognostic model for critically ill patients in resource limited settings in South Asia. Crit Care. 2017;21(1):1-8.

41. Lie KC, Lau CY, Van Vinh CN, West TE, Limmathurotsakul D, Sudarmono P, et al. Utility of SOFA score, management and outcomes of sepsis in Southeast Asia: a multinational multicenter prospective observational study. J Intensive Care. 2018;6(1):1-8.

42. Edward U, Sawe HR, Mfinanga JA, Ottaru TA, Kiremeji M, Kitapondya DN, et al. The utility of point of care serum lactate in predicting serious adverse outcomes among critically ill adult patients at urban emergency departments of tertiary hospitals in Tanzania. Trop Med Health. 2019;47(1): $1-13$.

43. Rello J, Leblebicioglu H. Sepsis and septic shock in low-income and middleincome countries: need for a different paradigm. Int J Infect Dis. 2016;48: $120-2$.

44. Drain PK, Hyle EP, Noubary F, Freedberg KA, Wilson D, Bishai WR, et al. Diagnostic point-of-care tests in resource-limited settings. Lancet Infect Dis. 2014;14(3):239-49.

45. Semret M, Ndao M, Jacobs J, Yansouni CP. Point-of-care and point-of-'can': leveraging reference-laboratory capacity for integrated diagnosis of fever syndromes in the tropics. Clin Microbiol Infect. 2018;24(8):836-44.

\section{Publisher's Note}

Springer Nature remains neutral with regard to jurisdictional claims in published maps and institutional affiliations.

Ready to submit your research? Choose BMC and benefit from:

- fast, convenient online submission

- thorough peer review by experienced researchers in your field

- rapid publication on acceptance

- support for research data, including large and complex data types

- gold Open Access which fosters wider collaboration and increased citations

- maximum visibility for your research: over $100 \mathrm{M}$ website views per year

At $\mathrm{BMC}$, research is always in progress.

Learn more biomedcentral.com/submissions 